\title{
The influence of dark matter halo onto evolution of supermassive black hole
}

\author{
M. I. Zelnikov* \\ E. A. Vasiliev ${ }^{\dagger}$ \\ I.E.Tamm Theoretical Department \\ Lebedev Physical Institute \\ Leninsky pr.53, Moscow, Russia
}

\begin{abstract}
The influence of dark matter (DM) on the growth of supermassive black holes (SMBHs) is studied. It is shown that gravitational scattering of DM particles on bulge stars leads to diffusion of DM in phase space $\left\{m, m_{z}, I\right\}$ ( $m$ denotes the angular momentum and $I$ is the radial action). Appropriate diffusion coefficients are calculated for different bulge models, and it is argued that the diffusion along $m$ axis is the most important effect. It is shown that this process leads to noticeable flow of DM into the black hole $(\mathrm{BH})$, resulting in its power-law growth: $M_{b h} \propto t^{9 / 16}$. Comparison with observational data shows that, in principle, this effect may explain observed masses of SMBHs. Special attention is paid to the corrections related to the innermost region of $\mathrm{BH}$ gravitational influence and the diffusion along $I$ axis. Their influence on the BH growth law is shown to be negligible.
\end{abstract}

\section{Introduction}

The interaction of a supermassive black hole in a galaxy center with dark matter halo is already much investigated 123 . There an adiabatic invariant approach is applied for different halo structure. The initial halo profiles are taken to be self-similar (power-law) $\rho \sim r^{-\alpha}(0<\alpha<2)$, isothermal $\left(\rho \sim r^{-2}\right)$ and NFW profiles 4 ( $\rho \sim$ $\left.\frac{\delta_{c}}{r / r_{s}\left(1+r / r_{s}\right)^{2}}\right)$. In Ref. [1 the method of adiabatic invariant is used to calculate small changes in orbital parameters of particles caused by slow variation of gravitational potential due to the black hole growth. This approach has two drawbacks: absorption by $\mathrm{BH}$ is not taken into account, and appropriate change of DM distribution function is neglected. The latter means that the loss cone is always full, thus leading to overestimation of dark matter flow.

More correct approach is used in Refs. [5, 6. There the evolution of DM distribution function due to absorption by $\mathrm{BH}$ and changes in $\mathrm{BH}$ mass and loss cone parameters are considered consistently. The authors come to the conclusion that for current values of black hole masses the fraction of dark matter inside them is rather small.

*zelnikov@lpi.ru

†eugvas@lpi.ru 
This approach was developed in Ref. 7] where the change of dark matter distribution function outside the loss cone due to diffusion in phase space was taken into account. It was demonstrated that this diffusion caused by gravitational scattering of DM particles on stars effectively refills the loss cone and determines the $\mathrm{BH}$ growth law. This effect was shown to give reasonable estimate for observed black holes masses.

The present work further develops the mentioned approach by accounting for three other factors: change of gravitational potential in the vicinity of black hole, modification of star distribution in this region, and the conditions under which the diffusion becomes effectively two-dimensional.

The paper is organized as follows. In the first section we introduce our model of dark matter halo and the galactic bulge. In the second section we write down the kinetic equation which describes the evolution of dark matter halo in phasespace, and calculate several quantities related to star distribution and dark matter motion. Calculation of the diffusion coefficients is performed in the third section. Also estimated are the diffusion timescales and corrections related to the central region. In the fourth section the solution of the diffusion equation in one-dimensional form is presented, the black hole growth law is established, and the corrections to the DM flow due to two-dimensional diffusion and the central region are investigated. It is shown that these corrections in most cases play no significant role in the diffusion process. Finally, in the section 5 we present some numerical results and discuss them.

\subsection{Dark matter in galaxies}

While the most part of matter in the Universe is now proved to be dark, the nature of the dark matter still remains unclear. In most models, however, the significant or dominant part of dark matter is taken to consist of cold dark matter (CDM), i.e. non-relativistic particles interacting only gravitationally. This leads to the absence of thermodynamical equilibrium in DM gas and the necessity of kinetic approach. Following this approach it was shown that the initial inhomogenities in DM distribution grow and form spherically-symmetric structures - non-dissipative gravitational singularities (NGSs) of different scales, having the same internal structure. General

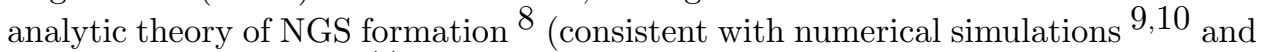
other analytic theories 11) predicts that all these structures have similar density profiles described by the following formula:

$$
\rho(r)=A r^{-\xi}, \xi=12 / 7 .
$$

The density profiles of DM haloes appearing in numerical simulations (see Refs. 4, 10) have different forms, typically shallower in the centre. While being more realistic in the outer parts than the profile (11) due to hierarchical formation mechanism, they cannot describe well the very inner region because of limited spatial resolution, thereby allowing different values of the inner slope 1214 ranging from -1 to -1.5 , 
all of which are within the errors in the simulations. Furthermore, observations do not exclude cuspy density profiles 13, but still are unable to determine the inner slope precisely. Therefore, the adopted profile does not contradict observations and is convenient for a number of reasons (simplicity of distribution function and tight relation between angular momentum and energy).

As initial inhomogenities are not spherically symmetric, the particles of NGS possess angular momenta (though total angular momentum of NGS is zero). High degree of contraction at the nonlinear stage makes these momenta relatively small compared to maximal possible angular momenta for corresponding orbital sizes. It turns out that angular momentum of a particle is related to its radial action $I$ by the formula: $m^{2}=l_{0}^{2} I^{2}$, where $I=\frac{1}{\pi} \int_{r_{-}}^{r_{+}} v_{r}(r) d r, v_{r}=\sqrt{2(E-\Psi(r))-\frac{m^{2}}{r^{2}}}$ is the radial velocity, $\Psi(r)$ is the gravitational potential, $r_{ \pm}$are the turnpoints, and $l_{0} \simeq 0.1$ is a small parameter.

In this case, the distribution function of DM in canonical variables $I, m, m_{z}$ has the following form:

$$
f\left(I, m, m_{z}\right)=f_{0} I^{1 / 8} \delta\left(m^{2}-l_{0}^{2} I^{2}\right)
$$

The average value of $m$ is zero, though the average $m^{2}$ is nonzero.

The formation of NGS of galactic scale is followed by the formation of galaxy itself due to infall of baryonic matter into gravitational well of NGS, its subsequent cooling and formation of galactic structures - disc, bulge and halo. After this stage the total gravitational potential is no longer defined by DM; conversely, in the central region it is mainly directed by baryons. Galaxy formation process is slow enough as compared to the dynamical time of DM particles, so that the gravitational potential evolution is adiabatic. This fact ensures that radial action is integral of motion (adiabatic invariant). It is defined by equation

$$
I(E, m)=\frac{1}{\pi} \int_{r_{-}}^{r_{+}} d r \sqrt{2(E-\Psi(r))-\frac{m^{2}}{r^{2}}}
$$

where $r_{-}, r_{+}$are minimal and maximal distances of a DM particle from center; $r_{-} \ll r_{+}$due to small angular momentum $(m \ll I)$.

Our main task is to study the central region of a galaxy - the bulge, which we may consider to be spherically symmetric. Under this assumption angular momentum is also conserved, and we obtain that the DM distribution function written in variables $I, m, m_{z}$ does not depend on time. Spatial distribution of DM is given by relation

$$
\rho(r)=(2 \pi)^{3} \frac{1}{4 \pi r^{2}} \int_{0}^{\infty} d m \int_{-m}^{+m} d m_{z} \int_{\Omega} d E \frac{\sqrt{2}}{\pi} \frac{1}{2 \sqrt{E-\Psi-\frac{m^{2}}{2 r^{2}}}} f(E, m),
$$

$\Omega$ is the energy interval where the expression under the radical sign is non-negative.

Let us assume that the density and the potential of the bulge have power-law profiles:

$$
n(r) \propto r^{-(2-\alpha)}, \quad M_{b}(r) \propto r^{\alpha+1}, \quad \Psi(r) \propto r^{\alpha},
$$


Then one can show 51$]$ that the radial action may be approximated (with the accuracy better than $8 \%$ ) in a factorised form

$$
\begin{aligned}
& I=I_{0}\left(r_{+}\right) C(\mu), \quad \mu=\frac{m}{I_{0}} \\
& I_{0}=\sqrt{G M_{b}\left(r_{+}\right) r_{+}} \\
& C(\mu)=\frac{\sqrt{2}}{\pi} \int_{\frac{r_{-}}{r_{+}}}^{1} d \chi\left[\int_{\chi}^{1} \xi^{\alpha-1} d \xi-\frac{\mu^{2}}{2}\left(\frac{1}{\chi^{2}}-1\right)\right]^{1 / 2}
\end{aligned}
$$

where function $C(\mu)$ at small argument $\mu$ is linear:

$$
C(\mu) \approx C(0)-b_{\alpha} \mu, \quad C(0)>0, \quad b_{\alpha} \sim 1
$$

Hence the DM density profile is also power-law:

$$
\rho(r)=A^{\prime} r^{-\xi^{\prime}}, \quad \xi^{\prime}=-\frac{15}{8}+\frac{9}{16} \alpha
$$

Let us now overview the bulge structure and proceed to estimation of DM fraction in the bulge.

\subsection{Bulge overview}

Bulge is the central part of a galaxy, for our Galaxy its radius being about $1 \mathrm{kpc}$ and mass about $10^{10} M_{\odot}$ 15. We assume it to be spherically symmetric. Bulge density profile can be derived from the dependency of stars velocity dispersion on distance to galactic center; we take it to be power-law: $n(r)=\tilde{\eta}_{b} r^{-(2-\alpha)}$. For isothermal bulge, i.e. having uniform velocity dispersion, $\alpha=0$. Milky Way bulge may be approximated with velocity dispersion $\sigma \propto r^{1 / 4}$ for $r \leq 50 \mathrm{pc}$, which corresponds $\alpha=0.5$.

It should be noticed that currently available observations of distant galaxies usually do not have enough resolution to derive velocity dispersion on scales less than a hundred parsecs 16. We assume their bulges to be isothermal in central parts, but one can show that the exact form of density profile does not matter much; only its integral characteristics affect the final result.

Generally, in the center of a galaxy a compact object is located, which is assumed to be a supermassive black hole (for a recent review see Ref. 17). For our own Galaxy it is now proved that the compact object Sgr A* is a black hole with mass $M_{b h} \simeq 2.9 \cdot 10^{6} M_{\odot}[18$. The innermost star cluster has the following density profile:

$$
n(r)=n_{0}\left(\frac{r}{r_{0}}\right)^{-3 / 2} \text {, where } n_{0}=10^{8} \frac{1}{\mathrm{pc}^{3}}, r_{0}=0.02 \mathrm{pc} 19 \text {. }
$$

Hence the mass of this cluster is $M(r)=\int_{0}^{r} M_{s} n\left(r^{\prime}\right) 4 \pi r^{\prime 2} d r^{\prime}=$ $\frac{8 \pi}{3} M_{s} n_{0} r_{0}{ }^{3}\left(\frac{r}{r_{0}}\right)^{3 / 2}$. For the sake of simplicity we have taken the masses of all 
stars to be equal $M_{s}=M_{\odot}$. Let us define the influence radius $R_{0}$ of $\mathrm{BH}$ by the condition that the total mass of stars inside this radius equals that of the $\mathrm{BH}$ :

$$
R_{0}=\left(\frac{M_{b h}}{M_{s}} \frac{3}{8 \pi n_{0} r_{0}^{3 / 2}}\right)^{2 / 3} \approx 1 \mathrm{pc} \text { at the moment. }
$$

So we may assume that inside this radius the gravitational potential is dominated by $\mathrm{BH}$, while outside it the influence of $\mathrm{BH}$ is negligible.

Notice that $\mathrm{BH}$ and bulge evolution changes values of $n_{0}$ and $R_{0}$. If we assume that the star density is continuous at $R_{0}$ while $R_{0}$ increases due to $\mathrm{BH}$ growth, then the following relation between $M_{b h}$ and $R_{0}$ arises:

$$
M_{b h}=\frac{8 \pi}{3} M_{s} \tilde{\eta}_{b} R_{0}{ }^{1+\alpha} .
$$

Here $\tilde{\eta}_{b}$ is the coefficient in the bulge density profile; for isothermal bulge with velocity dispersion $\sigma_{0} \tilde{\eta}_{b}=\frac{\sigma_{0}{ }^{2}}{2 \pi G M_{s}}($ see (35) $)$.

\subsection{Dark matter in the bulge}

The total mass of Milky Way's dark matter halo can be assessed to be $M_{H} \sim$ $10^{12} M_{\odot}$, its radius to be $R_{H} \sim 100 \mathrm{kpc} 21$. From (5) we derive adiabatic invariant for particles with $R_{+}=R_{H}$ to be $I_{\max }=0.35\left(G M_{H} R_{H}\right)^{1 / 2}$. If we suppose that the initial distribution (2) can be extrapolated up to $R_{+}=R_{H}$, then tha total halo mass is

$$
M_{H}=\frac{8}{9}(2 \pi)^{3} f_{0} I_{\max }^{9 / 8}
$$

and hence

$$
f_{0}=3.5 \frac{1}{(2 \pi)^{3}} \frac{M_{H}{ }^{7 / 16}}{\left(G R_{H}\right)^{9 / 16}} .
$$

Then the value of $f_{0}$ for our Galaxy is $6 \cdot 10^{8} \mathrm{~g}\left(\frac{\mathrm{cm}^{2}}{\mathrm{~s}}\right)^{-9 / 8}$.

In this case the relative amount of DM inside the bulge is given by

$$
\Upsilon=\frac{M_{D M}}{M_{B}}=\frac{M_{H}}{\left(G M_{H} R_{H}\right)^{9 / 8}} \frac{\left(G M_{B} R_{B}\right)^{9 / 8}}{M_{B}}=\left(\frac{M_{H}}{M_{B}}\right)^{\frac{7}{16}}\left(\frac{R_{B}}{R_{H}}\right)^{\frac{9}{16}} \sim 1 .
$$

This means that for the model distribution (2) the mass of dark matter inside bulge is comparable to the baryonic mass. The same relation is valid in the innermost region (BH domain of influence). However, the distribution (21) is applicable only in some vicinity of the galactic center, and hence the actual values of $f_{0}$ and $\Upsilon$ can deviate from the assessments 12 13).

Observations and modelling of mass distribution in the Galaxy do not give tight restrictions on the amount of DM in the bulge. Depending on the model, $\Upsilon$ may comprise from $1 / 4$ to $1 / 3$ of the total mass inside the central $3 \mathrm{kpc}$ region 13 , but not the major fraction of the bulge mass. So, for the final calculations of the $\mathrm{BH}$ mass we should decrease the value of $f_{0}(12)$ by a factor of $3-4$ or more. 


\section{Problem definition and particle motion parameters}

Our goal is to determine the possible fraction of $\mathrm{DM}$ in $\mathrm{BH}$, and to derive the growth law of BH due to absorption of DM. Firstly, one can prove that the direct capture of particles with momenta less than $m_{g}=\frac{4 G M_{b h}}{c}$ does not lead to significant growth of $\mathrm{BH}$ since their mass calculated from distribution (2) with current value of $m_{g}$ is several orders of magnitude less than $M_{b h}[$.

The simplest process which changes the distribution function of DM is gravitational scattering of DM particles on stars identical to Coulomb scattering in plasma.

\subsection{Kinetic equation}

The dark matter distribution function satisfies the following equation

$$
\frac{\partial f(\vec{r}, \vec{v}, t)}{\partial t}+\left\{H_{0}, f\right\}=S t\{f\}
$$

where $H_{0}$ is the Hamilton function for gravitational interaction, $\{$,$\} is the Poisson$ bracket, $S t\{f\}$ is the collision term in Landau form 22 .

$$
\begin{aligned}
& S t\{f\}=\frac{\partial}{\partial\left(\mu v_{i}\right)} \int\left[f(\vec{v}) \frac{\partial f^{\prime}\left(\vec{v}^{\prime}\right)}{\partial\left(\mu v_{j}^{\prime}\right)}-f^{\prime}\left(\vec{v}^{\prime}\right) \frac{\partial f(\vec{v})}{\partial\left(\mu v_{j}\right)}\right] B_{i j} d^{3} v^{\prime}, \\
& B_{i j}=\frac{2 \pi G^{2} \mu^{\prime 2} \mu^{2} L_{c}}{|u|}\left(\delta_{i j}-\frac{u_{i} u_{j}}{u^{2}}\right) .
\end{aligned}
$$

Here entities with primes refer to stars and other to DM particles; masses are denoted as $\mu, \mu^{\prime}$ to avoid confusion with momentum. $\vec{u}=\vec{v}-\vec{v}^{\prime}$ is the relative velocity, $L_{c} \sim 15$ is the Coulomb logarithm. Since the mass of a star $M_{s} \equiv \mu^{\prime} \gg \mu$, we neglect the first term in equation:

$$
\begin{aligned}
& S t\{f\}=\frac{\partial}{\partial v_{i}}\left[W_{i j} \frac{\partial f}{\partial v_{j}}\right], \\
& W_{i j}=2 \pi G^{2} M_{s}^{2} L_{c} \int \frac{u^{2} \delta_{i j}-u_{i} u_{j}}{u^{3}} f^{\prime}\left(\vec{v}^{\prime}, r\right) d^{3} v^{\prime} .
\end{aligned}
$$

As soon as collision frequency of DM particles with stars is much less than their orbital motion frequency, one can rewrite the equation (14) in the form averaged over a period in action-angle variables $\left\{I_{k}, \phi_{k}\right\} ;\left\{I_{k}\right\}=\left\{I, m, m_{z}\right\}$ :

$$
\frac{\partial f\left(\left\{I_{k}\right\}, t\right)}{\partial t}=S t\{f\} \text {. }
$$

Then the equation (16) for collision term looks as follows:

$$
\begin{aligned}
& S t\{f\}=\frac{\partial}{\partial I_{k}}\left[R_{k l} \frac{\partial f}{\partial I_{l}}\right], \\
& R_{k l}=\frac{1}{(2 \pi)^{3}} \int d^{3} \phi \frac{\partial I_{k}}{\partial v_{i}} \frac{\partial I_{l}}{\partial v_{j}} W_{i j} .
\end{aligned}
$$

The star distribution around a SMBH and their diffusion towards the disruption boundary was studied in Refs. 23, 24 Two-dimensional Fokker-Planck equation was 
written in coordinates $E$ and $m$, and solved analytically with certain simplifications, introducing the notion of energy-dependent loss-cone. A fraction of stars with small angular momenta is eliminated during one dynamical time, and the diffusion along energy axis leads to capture of stars with critical (minimal) energy. We use radial action $I$ instead of energy, because diffusion along $I$ axis does not lead to capture; therefore the absorption process looks more clear. Also, our distribution function is time-dependent and the black hole mass changes in time.

Observations show that the distribution function (DF) of stars may be considered as isotropic, i.e. not depending on momentum, even in the central region 18 . For isotropic DF the tensor $W_{i j}$ takes the following form 7 .

$$
\begin{gathered}
W_{i j}=A(E, r) \delta_{i j}-B(E, r) \frac{v_{i} v_{j}}{v^{2}}, \\
A=\frac{16 \pi^{2}}{3} G^{2} M_{s}^{2} L_{c} \int_{\Psi(r)}^{\infty} d E^{\prime} f^{\prime}\left(E^{\prime}\right) \begin{cases}1 & , E \leq E^{\prime} \\
\frac{3}{2} \frac{v^{\prime}}{v}\left(1-\frac{v^{\prime 2}}{3 v^{2}}\right), E>E^{\prime}\end{cases} \\
A-B=\frac{16 \pi^{2}}{3} G^{2} M_{s}^{2} L_{c} \int_{\Psi(r)}^{\infty} d E^{\prime} f^{\prime}\left(E^{\prime}\right) \begin{cases}1 & , E \leq E^{\prime} \\
\left(\frac{v^{\prime}}{v}\right)^{3}, & E>E^{\prime} .\end{cases}
\end{gathered}
$$

From (56) it follows that adiabatic invariant may be represented as follows:

$$
I(E, m) \approx J(E)-b_{\alpha} m .
$$

Note that in the case of Coulomb $(\alpha=-1)$ and oscillator $(\alpha=2)$ potential the constant $b_{\alpha}=1$, and in the case of isothermal potential (or close to it) $b_{\alpha} \approx 0.6$.

It is convenient to perform linear variable change: $\left\{I, m, m_{z}\right\} \rightarrow\left\{J, m, m_{z}\right\}$ Since it is linear, the expression (19) for tensor $R_{k l}$ does not change. In addition, due to small value of parameter $l_{0}$, the initial distribution function (2) has the same form in new variables:

$$
f_{i}(J, m)=f_{0} J^{1 / 8} \delta\left(m^{2}-l_{0}^{2} J^{2}\right)
$$

The initial distribution function does not depend on $m_{z}$, hence the solution of kinetic equation (17) will not depend on $m_{z}$ neither. We rewrite the expression (18) for collision term as follows:

$$
S t\{f\}=\frac{1}{m} \frac{\partial}{\partial m} m\left(R_{22} \frac{\partial f}{\partial m}+R_{12} \frac{\partial f}{\partial J}\right)+\frac{\partial}{\partial J}\left(R_{12} \frac{\partial f}{\partial m}+R_{11} \frac{\partial f}{\partial J}\right),
$$

where the diffusion coefficients (19) are the following 7 .

$$
\begin{aligned}
& R_{11}=\left(\frac{\partial J}{\partial E}\right)^{2}\left\langle(A-B) v^{2}\right\rangle, \\
& R_{12}=\left(\frac{\partial J}{\partial E}\right)\langle(A-B) m\rangle, \\
& R_{22}=\left\langle A r^{2}-B \frac{m^{2}}{v^{2}}\right\rangle .
\end{aligned}
$$


Here the averaging over angle variables is defined as

$$
\begin{aligned}
& \langle X\rangle=\frac{2}{T} \int_{r_{-}}^{r_{+}} \frac{d r}{v_{r}} X, \\
& T=2 \int_{r_{-}}^{r_{+}} \frac{d r}{v_{r}}, \quad v_{r}=\sqrt{2(E-\Psi(r))-\frac{m^{2}}{r^{2}}},
\end{aligned}
$$

where $T$ is the particle oscillation period, $v_{r}$ is its radial velocity.

Before proceeding to the solution of the kinetic equation (17. 25) one should define the distribution function of stars.

\subsection{Distribution of stars}

We assume that the density profile of stars in the bulge is power-law: $n(r)=$ $n_{0}\left(\frac{r}{r_{0}}\right)^{-\gamma}$, and the gravitational potential is also power-law $\Psi(r)=\Psi_{0} r^{\alpha}$. In this case isotropic distribution function of stars can be written as a power-law dependence on energy 28 .

$$
\begin{gathered}
f^{\prime}\left(v^{\prime}, r\right)=F_{0} E^{-\beta}, \quad E=\frac{v^{\prime 2}}{2}+\Psi(r), \\
n(r)=\int_{0} f^{\prime}\left(v^{\prime}, r\right) d^{3} v^{\prime}=\int_{0}^{\infty} F_{0}\left(\frac{v^{\prime 2}}{2}+\Psi_{0} r^{\alpha}\right)^{-\beta} 4 \pi v^{\prime 2} d v^{\prime}= \\
=\frac{\Gamma\left(\beta-\frac{3}{2}\right)}{\Gamma(\beta)}(2 \pi)^{3 / 2} F_{0} \Psi_{0}^{\frac{3}{2}-\beta} r^{\alpha\left(\frac{3}{2}-\beta\right)}, \text { hence } \gamma=\left(\beta-\frac{3}{2}\right) \alpha .
\end{gathered}
$$

We consider two particular cases: a) self-consistent potential of stars in the bulge (from observations 25 it follows that the star distribution in the bulge is close to isothermal, so we take the power-law index $\gamma$ close to 2) and b) the central region of bulge where potential is dominated by the black hole.

In the first case we have

$$
\begin{gathered}
\frac{d \Psi(r)}{d r}=\frac{4 \pi G M(r)}{r^{2}}=\frac{4 \pi}{3-\gamma} G M_{s} n_{0} r_{0}^{\gamma} r^{1-\gamma}, \text { hence } \\
\Psi=\Psi_{0} r^{\alpha}, \quad \Psi_{0}=\frac{4 \pi G M_{s} n_{0} r_{0}^{\gamma}}{(3-\gamma)(2-\gamma)}, \quad \gamma=2-\alpha, \\
\sigma=\sigma_{0} r^{\alpha / 2} \text { is the velocity dispersion, } \sigma_{0}^{2}=\Psi_{0} \frac{\alpha}{2(1-\alpha)}, \\
f^{\prime}(E)=F_{0} E^{-\beta}, \quad F_{0}=\frac{\alpha(\alpha+1)}{4 \pi G M_{s}(2 \pi)^{3 / 2}} \frac{\Gamma(\beta)}{\Gamma\left(\beta-\frac{3}{2}\right)} \Psi_{0}{ }^{\frac{2}{\alpha}}, \quad \beta=\frac{2}{\alpha}+\frac{1}{2} .
\end{gathered}
$$


In the limit $\alpha \rightarrow 0$ these expressions describe isothermal star cluster. Let us rewrite them in this particular case separately:

$$
\begin{aligned}
& \Psi(r)=\Psi_{0} \ln \frac{r}{r_{0}}, \quad \Psi_{0}=2{\sigma_{0}}^{2}, \quad \sigma_{0} \text { is the velocity dispersion, } \\
& n(r)=n_{0}\left(\frac{r}{r_{0}}\right)^{-2}, \quad n_{0} r_{0}^{2}=\frac{\sigma_{0}^{2}}{2 \pi G M_{s}} \\
& f^{\prime}(E)=F_{0} \exp \left(-\frac{E}{\sigma_{0}^{2}}\right), \quad F_{0}=n_{0}\left(2 \pi{\sigma_{0}}^{2}\right)^{-3 / 2} .
\end{aligned}
$$

In the second case the particle dynamics is governed by the Coulomb potential of the black hole.

$$
\Psi(r)=-\frac{G M_{b h}}{r}
$$

Restricting our consideration to the case $\gamma=3 / 2$ which follows from observations 19, we obtain from (30) $\beta=0$. Hence the distribution function does not depend on energy and equals $F_{0}$ at $E<0$ :

$$
f^{\prime}(E)=F_{0}=\frac{3}{8 \sqrt{2} \pi\left(G M_{b h}\right)^{3 / 2}} \frac{3 M_{b h}}{8 \pi M_{s} R_{0}^{3 / 2}},
$$

where $M_{b h}$ and $R_{0}$ are related by (10).

\subsection{Dark matter motion parameters}

In this section we present the motion parameters for DM particles for practically important cases.

$$
\begin{aligned}
I(E, m) & =\frac{1}{\pi} \int_{r_{-}}^{r_{+}} \sqrt{2(E-\Psi(r))-\frac{m^{2}}{r^{2}}} d r-\text { adiabatic invariant; } \\
I(E, 0) & \equiv J(E), \quad r_{ \pm}-\text {turnpoints; } \\
T(E, m) & =2 \pi \frac{\partial I}{\partial E}-\text { particle oscillation period; } \\
\frac{r_{-}}{r_{+}} & =\chi_{\min } \ll 1, \quad \frac{m}{J}=\mu \ll 1 .
\end{aligned}
$$

For power-law potential (31):

$$
\begin{aligned}
J(E) & =C_{\alpha} \frac{\sigma_{0} r_{+}{ }^{1+\frac{\alpha}{2}}}{\sqrt{\pi}}, \quad C_{\alpha}=\frac{1}{\sqrt{\pi}} \int_{0}^{1} d \chi \sqrt{\frac{4(1-\alpha)}{\alpha}\left(1-\chi^{\alpha}\right)} \approx 1-0.8 \alpha, \\
T(E) & =C_{\alpha} \frac{1+\frac{\alpha}{2}}{1-\alpha} \frac{\sqrt{\pi} r_{+}{ }^{1-\frac{\alpha}{2}}}{\sigma_{0}} \\
r_{+} & =\left(\frac{E}{2 \sigma_{0}^{2}} \frac{\alpha}{1-\alpha}\right)^{1 / \alpha}, \quad \chi_{\min }=C_{\alpha} \sqrt{\frac{\alpha}{4 \pi(1-\alpha)}} \mu
\end{aligned}
$$


In the limit $\alpha \rightarrow 0$ we obtain formulas for isothermal potential (34):

$$
\begin{aligned}
J(E) & =\frac{1}{\sqrt{\pi}} \sigma_{0} r_{+}, \\
T(E) & =\sqrt{\pi} r_{+} / \sigma_{0}, \\
r_{+} & =r_{0} \exp \left(\frac{E}{2 \sigma_{0}^{2}}\right), \quad \chi_{m i n}=\frac{0.25 \mu}{\sqrt{1.3-\ln \mu}} .
\end{aligned}
$$

For Coulomb potential (37):

$$
\begin{aligned}
J(E) & =\frac{1}{\sqrt{2}} \sqrt{G M_{b h} r_{+}}, \\
T(E) & =\frac{\pi}{\sqrt{2}} \frac{G M_{b h}}{|E|^{3 / 2}}, \\
r_{+} & =\frac{G M_{b h}}{|E|}, \quad \chi_{\min }=\frac{\mu^{2}}{2} .
\end{aligned}
$$

\section{The diffusion coefficients}

Two opposite cases should be distinguished: for particles with apocenter distances $r_{+}(J)<R_{0}$ ( $R_{0}$ is the radius of BH's domain of influence (10) ) we calculate coefficients using Coulomb potential, and for other particles we use the expressions for

self-consistent bulge potential (this corresponds to assumption that these particles spend the most part of orbital period outside BH's domain of influence).

\subsection{The diffusion coefficients for bulge}

Below we present the quantities $A, A-B$ for power-law (including isothermal) star distribution:

$$
\begin{aligned}
A & =\frac{4}{3 \sqrt{2 \pi}} G M_{s} L_{c} \sigma(r) r^{-2} \cdot \widetilde{A}_{\alpha}(\tilde{v}), \quad \tilde{v}=\frac{v(r)}{\sigma(r)}, \\
A-B & =\frac{4}{3 \sqrt{2 \pi}} G M_{s} L_{c} \sigma(r) r^{-2} \cdot \widetilde{\Omega}_{\alpha}(\tilde{v}),
\end{aligned}
$$

where

$$
\begin{aligned}
\widetilde{A}_{\alpha}(\tilde{v}) & =\left[\frac{\left(\tilde{v}^{2} / 2 \varsigma+1\right)^{-(\beta-1)}}{\beta-1}+\frac{\tilde{v}^{2}}{2 \varsigma} F\left(\frac{3}{2}, \beta ; \frac{5}{2} ;-\frac{\tilde{v}^{2}}{2 \varsigma}\right)-\frac{\tilde{v}^{2}}{10 \varsigma} F\left(\frac{5}{2}, \beta ; \frac{7}{2} ;-\frac{\tilde{v}^{2}}{2 \varsigma}\right)\right] \times \\
& \times \frac{\alpha(\alpha+1) \sqrt{\varsigma}}{2} \frac{\Gamma(\beta)}{\Gamma\left(\beta-\frac{3}{2}\right)}, \\
\widetilde{\Omega}_{\alpha}(\tilde{v}) & =\left[\frac{\left(\tilde{v}^{2} / 2 \varsigma+1\right)^{-(\beta-1)}}{\beta-1}+\frac{\tilde{v}^{2}}{5 \varsigma} F\left(\frac{5}{2}, \beta ; \frac{7}{2} ;-\frac{\tilde{v}^{2}}{2 \varsigma}\right)\right] \times \frac{\alpha(\alpha+1) \sqrt{\varsigma}}{2} \frac{\Gamma(\beta)}{\Gamma\left(\beta-\frac{3}{2}\right)}, \\
\varsigma & =2 \frac{1-\alpha}{\alpha}=\frac{\Psi_{0}}{\sigma_{0}^{2}}, \quad F(. . . . . . . ; .) \text { is the hypergeometric function, }
\end{aligned}
$$


and for isothermal profile

$$
\begin{array}{ll}
\widetilde{A}_{0}(\tilde{v})=\frac{3}{2}\left[\frac{\exp \left(-\frac{\tilde{v}^{2}}{2}\right)}{\tilde{v}^{2}}+\frac{\tilde{v}^{2}-1}{\tilde{v}^{3}} \Phi(\tilde{v})\right], & \\
\widetilde{\Omega}_{0}(\tilde{v})=3\left[-\frac{\exp \left(-\frac{\tilde{v}^{2}}{2}\right)}{\tilde{v}^{2}}+\frac{1}{\tilde{v}^{3}} \Phi(\tilde{v})\right], & \begin{array}{l}
\Phi(x)=\int_{0}^{x} \exp \left(-\frac{t^{2}}{2}\right) d t \\
\text { is the probability integral. }
\end{array}
\end{array}
$$

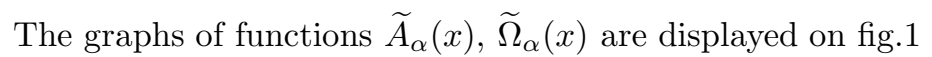

Now let us calculate the diffusion coefficients $R_{11}, R_{12}, R_{22}$ according to (26).

First of all, we are interested in low values of momentum, so we neglect the second term in $R_{22}$, which is $\sim \mu^{2}$ times smaller than the first one.

$$
\begin{gathered}
R_{22}=\frac{8}{3 \sqrt{2 \pi}} G M_{s} L_{c} r_{+} T^{-1} \int_{\chi_{\text {min }}}^{1} \frac{d \chi}{\tilde{v}} \widetilde{A}(\tilde{v}), \quad \chi=\frac{r}{r_{+}} \\
\tilde{v}= \begin{cases}\sqrt{\frac{4(1-\alpha)}{\alpha}} \sqrt{\chi^{-\alpha}-1}, & \alpha>0 \\
\sqrt{-4 \ln (\chi)} & , \alpha=0 .\end{cases}
\end{gathered}
$$

We take the lower limit of integration in $\chi_{\min }$ to be zero, since at low $\chi$ (high $\tilde{v}$ ) the integrand is small. Then we have for $R_{22}$ the following expression:

$$
R_{22} \approx 0.46 G M_{s} L_{c} \sigma_{0}^{\frac{1}{1+\alpha / 2}} J^{\frac{\alpha}{2+\alpha}} .
$$

This conforms the value obtained in Ref. 7 Note that $R_{22}$ does not depend on $m$ and weakly depends on $J$, not depending on $J$ at all in isothermal case.

For $R_{11}$ we have

$$
R_{11}=\frac{8}{3 \sqrt{2 \pi}} G M_{s} L_{c} r_{+} T^{-1}\left(\frac{T \sigma_{0} r_{+}{ }^{\alpha / 2-1}}{2 \pi}\right)^{2} \int_{\chi_{\min }}^{1} d \chi \chi^{-2+\alpha} \tilde{v} \widetilde{\Omega}(\tilde{v}) .
$$

The integral diverges at lower limit, so we take the value 411 for $\chi_{\min }$. For the isothermal case $(\alpha=0)$ it is possible to obtain an asymptotic approximation of this integral at low $\chi_{\text {min }}$. Since $\widetilde{\Omega}_{0}(\tilde{v}) \approx \frac{3 \sqrt{\pi}}{\sqrt{2} \tilde{v}^{3}}$, the integral approximately equals $\left(-\chi_{\min } \ln \chi_{\min }\right)^{-1} \sim 1 / \mu$. A similar consideration for $\alpha>0$ leads to an estimate of the integral as $\mu^{-1+2 \alpha}$. The expression in brackets before the integral weakly depends on $\alpha$ and equals $1 / 2 \sqrt{\pi}$ for $\alpha=0$. Then

$$
R_{11} \approx R_{22} \cdot 0.1\left(\frac{J}{m}\right)^{1-2 \alpha}
$$

The last coefficient $R_{12}$ can be represented as $R_{22} \cdot K_{\alpha}(\mu)$, where $K_{\alpha}(\mu) \rightarrow 0$ at $\mu \rightarrow 0$. So one can neglect the term with $R_{12}$ in (25) at low $\mu$. 


\subsection{The diffusion coefficients for central region}

We restrict the calculation to the case when the density of $\operatorname{stars} n(r) \propto r^{-3 / 2}$. Then

$$
R_{22}=\frac{32 \pi^{2}}{3 \sqrt{2}} G^{2} M_{s}{ }^{2} L_{c} F_{0} r_{+}{ }^{3}|E|^{1 / 2} T^{-1} \int_{\chi_{\min }}^{1} \frac{\chi^{2} d \chi}{\sqrt{\frac{1}{\chi}-1}}\left(\frac{4}{5 \chi}-\frac{1}{5}\right) .
$$

The integral weakly depends on $\chi_{\min }$ at low $\chi_{\min }$ and equals $\approx 1.14$. Finally, we have

$$
R_{22}=2.4 G^{1 / 2} M_{s} M_{b h}^{-1 / 2} R_{0}^{-3 / 2} L_{c} J^{2} .
$$

Comparing (54) with expression (51) for bulge, we conclude that they coincide at $J=J\left(R_{0}\right)$ (i.e. at the boundary of central region of the black hole influence). This means that the expressions for two limiting cases are consistent with each other.

$$
R_{11}=\frac{16}{3 \sqrt{2}} G^{2} M_{s}^{2} L_{c} F_{0} r_{+}|E|^{3 / 2} T \int_{\chi_{\min }}^{1} d \chi \sqrt{\frac{1}{\chi}-1}\left(\frac{2}{5 \chi}+\frac{3}{5}\right)
$$

Here the integral approximately equals $0.8 / \sqrt{\chi_{\min }}$; so with the aid of (47) we obtain

$$
R_{11}=R_{22} \cdot 0.25 \frac{\mathrm{J}}{\mathrm{m}}
$$

Similarly to the previous case we get that $R_{12} \simeq$ const $R_{22} \frac{m}{J}$, so it can be neglected at low $m$.

\subsection{Diffusion timescale estimate}

One can easily see that for an equation $\frac{\partial f}{\partial t}=\frac{\partial}{\partial x}\left(A \frac{\partial f}{\partial x}\right)$ the characteristic time of "equillibrium establishment" at spatial scale $l$ equals $\tau \sim \frac{l^{2}}{8 A}$. Let us make a few estimates for kinetic equation (17) with coefficients (51 [53) calculated for the bulge.

The diffusion timescale for momenta $\tau_{2}=\frac{\left(l_{0} J_{0}\right)^{2}}{8 R_{22}} \sim 10^{6}\left(\frac{r_{+}}{1 \mathrm{pc}}\right)^{2} \mathrm{yr}$, so that the galactic age corresponds to spatial area of $r_{+} \leq 100 \mathrm{pc}$. The coefficient $R_{11}$ increases with decreasing $m$. The diffustion timescale along $J$ axis at minimal momentum $m=m_{g}$ equals $\tau_{1} \sim 3 \cdot 10^{6} \frac{r_{+}}{1 \mathrm{pc}} \mathrm{yr}$, and for $r_{+} \sim 100 \mathrm{pc} \tau_{1}$ is much less that the galactic age. But for momenta at least an order of magnitude greater than $m_{g}$ the diffusion along $J$ axis does not disturb much the one-dimensional diffusion along $m$. We will show in the next section that if we account for a cutoff of star distribution function at $r \rightarrow 0$, then we obtain a finite limit for $R_{11}$ with decreasing $m$. Thus $\tau_{1}$ becomes comparable with $\tau_{2}$ or greater for all $r_{+} \leq 100 \mathrm{pc}$. Hence in the first approximation we can neglect the diffusion along $J$ in comparison with the diffusion along $m$, at least for not very small values of $m$.

In addition we determine the characteristic timescale for diffusion in the central region. Since $R_{22} \sim J^{2}=l_{0}^{2} m^{2}$ the timescale does not depent on initial value of momentum and equals $\tau_{2}=10^{6} \mathrm{yr} \times\left(\frac{M_{b h}}{3 \cdot 10^{6} M_{\odot}}\right)^{2}$. 


\subsection{Diffusion coefficients for an improved star distribution}

Indeed, in deriving relations (33) we have neglected the fact that number density of stars in the vicinity of black hole may be significantly less than that follows from general power-law profile due to stars capture or tidal disruption by black hole. From (52) we see that it is the stars in the pericenter of the orbit that affect the coefficient $R_{11}$ most of all. Hence for particles with low momenta these are the innermost stars, which number we may have overestimated. Now let us try to evaluate corrections linked to this fact.

Firstly, consider the Coulomb potential region. Observations show 20 that the nearest star's orbital axis approximately equals $1000 \mathrm{AU}=3 \cdot 10^{-4} \mathrm{pc}$. But from (8) we see that the number of stars inside a sphere of radius 1000 AU should be about 15 .

For correction we adopt that stars distribution function vanishes for energies $E<E_{c r}=-\frac{G M_{b h}}{r_{c r}}$. Thus the density profile (8) transforms to

$$
n(r)=n_{0}\left(\frac{r}{r_{0}}\right)^{-3 / 2}\left[1-\left(1-\frac{r}{r_{c r}}\right)^{3 / 2}\right] .
$$

Comparison with observations gives the value $r_{c r} \simeq 5 \cdot 10^{-3} \mathrm{pc} \sim 10^{4} r_{g}$.

Now let us calculate modified coefficient $R_{11}$. Following (55) we obtain the expression (with integration from 0 to 1 in this case):

$$
\begin{gathered}
R_{11}=\frac{16}{3 \sqrt{2}} G^{2} M_{s}^{2} L_{c} F_{0} r_{+}|E|^{3 / 2} T\left\{\begin{array}{l}
\left(\frac{3 \pi}{4}-\frac{\pi}{4} \chi_{c r}\right) / \sqrt{\chi_{c r}}, \chi_{\min }<\chi_{c r}<1 \\
\frac{\pi}{2 \chi_{c r}}, \chi_{c r}>1
\end{array}\right. \\
\chi_{c r}=\frac{r_{c r}}{r_{+}}
\end{gathered}
$$

For $r_{+}<1 \mathrm{pc}, \mu=\frac{m}{I} \leq 0.1$, we have $\chi_{c r}>\chi_{\min }$, hence in the whole central region of Coulomb potential the lower limit of integration $R_{11}$ is given by $\chi_{c r}$. This means that $R_{11}$ is in fact is much less (approximately $\frac{1}{3} \sqrt{\chi_{c r} / \chi_{\min }}$ times) than calculated from (56).

Secondly, the same formalism in the region of self-consistent (particularly, isothermal) potential should include the statement that distribution function of stars vanishes for sufficiently low energies. For isothermal star distribution this corresponds to well-known solution for isothermal sphere with core (i.e. no central cusp), and the core radius is of the same order that $r_{c r}$. Unfortunately, we can make no estimation for core radius from observations, since their spatial resolution is not enough. We may adopt the same value as calculated for our Galaxy $\left(r_{c r} \sim 10^{2} \div 10^{4} r_{g}\right)$. One can show that this assumption changes the lower limit of integration in (52) to value $\chi_{\min } \sim \max \left(r_{-}, r_{c r}\right) / r_{+}$. Thus $R_{11}$ increases with decreasing momentum up to $m=\left(10^{2} \div 10^{3}\right) m_{g}$ and then reaches constant limit. At the same time $R_{22}$ is not affected by this cut since the integrand in (50) is small at small $\chi$. 


\section{Dark matter absorption and growth of the black hole}

\subsection{One-dimensional diffusion approximation}

It was shown in previous sections that coefficient $R_{22}$ does not depend on $m$ in the bulge outside the central region of BH influence; coefficient $R_{11} \sim R_{22}\left(\frac{J}{m}\right)^{\epsilon}, \epsilon \leq 1$, and $R_{12} \sim R_{22} \frac{m}{J}$. Since at the initial moment $m=l_{0} J \ll J$ and our scope of interest lies in the domain of low momenta, we can take $m \ll J$ and disregard the term with $R_{12}$. Furthermore, from the same arguments it follows that $\frac{\partial f}{\partial J} \sim l_{0} \frac{\partial f}{\partial m}$ at the initial moment, and since $R_{11} \sim R_{22}$ at $m=l_{0} J$, then $R_{11} \partial f / \partial J \ll R_{22} \partial f / \partial m$ at not very low values of $m$. Finally, $f(m, J, t)=0$ at $m=m_{g}$, hence $\left.\frac{\partial f}{\partial J}\right|_{m=m_{g}}=0$, and $R_{11} \frac{\partial f}{\partial J}$ is limited at $m \rightarrow 0$. Also notice that in fact $R_{11}$ itself is limited at $m \rightarrow 0$, as noted previously.

To summarize, in the first approximation we leave in (25) only the first term and rewrite the kinetic equation (17) as follows:

$$
\frac{\partial f}{\partial t}=\frac{1}{m} R(J) \frac{\partial}{\partial m}\left(m \frac{\partial f}{\partial m}\right),
$$

with boundary conditions $\left.f\right|_{m=m_{g}}=0,\left.\quad m \frac{\partial}{\partial m} f\right|_{m=\infty}=0$,

initial conditions (12) and diffusion coefficient

$$
R(J)=0.46 G M_{s} L_{c} \sigma_{0}^{\frac{1}{1+\alpha / 2}} J^{\frac{\alpha}{2+\alpha}} \cdot\left\{\begin{array}{l}
1 \\
\left(\frac{J}{J_{0}}\right)^{2}, J>J_{0}\left(M_{b h}\right)
\end{array}\right.
$$

$$
\text { Here } J_{0}=\sqrt{G M_{b h} R_{0} / 2} \propto M_{b h} \frac{1+\alpha / 2}{1+\alpha}
$$

is the boundary value separating the $\mathrm{BH}$ domain of influence and bulge itself (as follows from (10, 45)).

The flux of dark matter through the surface $m=m_{g}$ is given by the expression

$$
\begin{aligned}
& S(t)=-(2 \pi)^{3} \iiint d J d m d m_{z} \frac{\partial f}{\partial t}=-(2 \pi)^{3} \iint d J d m 2 m \frac{1}{m} R \frac{\partial}{\partial m} m \frac{\partial f}{\partial m}= \\
& =\left.(2 \pi)^{3} \int d J \cdot 2\left(m R(J) \frac{\partial f}{\partial m}\right)\right|_{m=m_{g}}=2(2 \pi)^{3} \int d J f_{0} J^{1 / 8} S_{J}(t),
\end{aligned}
$$

where $S_{J}(t)=m_{g} R \frac{\partial f}{\partial m}$ is the flux through $m=m_{g}$ for equation (59) with initial condition

$$
f(m, t=0)=\delta\left(m^{2}-m_{0}^{2}\right), \quad m_{0}=l_{0} J
$$

Now we are going to calculate the time-dependent flux $S_{J}(t)$ of DM particles through the absorption boundary for each value of $J$ separately, i.e. one-dimensional flux, and then integrate it over $J$ assuming that the diffusion along $J$ is small. 


\subsection{Flux in one-dimensional diffusion}

Consider an auxilliary task: equation (59) with boundary conditions (60) and initial condition (64) and determine the flux $S_{J}(t)=m_{g} R \frac{\partial f}{\partial m}$.

The solution of eq.(59) may be represented as

$$
\begin{gathered}
f(m, t)=\int_{0}^{\infty} d m^{\prime} G\left(m, m^{\prime}, t\right) f\left(m^{\prime}, 0\right) \\
G=\int_{0}^{\infty} d \lambda m^{\prime} \exp (-\lambda R t) Z_{\lambda}\left(m, m_{g}\right) Z_{\lambda}\left(m^{\prime}, m_{g}\right) \text { is the Green function }, \\
Z_{\lambda}\left(m, m_{g}\right)=\frac{J_{0}\left(\sqrt{\lambda} m_{g}\right) Y_{0}(\sqrt{\lambda} m)-J_{0}(\sqrt{\lambda} m) Y_{0}\left(\sqrt{\lambda} m_{g}\right)}{\left(J_{0}^{2}\left(\sqrt{\lambda} m_{g}\right)+Y_{0}^{2}\left(\sqrt{\lambda} m_{g}\right)\right)^{1 / 2}}
\end{gathered}
$$

is the orthogonal system of fundamental functions of the boundary problem (60), $J_{0}, Y_{0}$ are Bessel functions of first and second kind of 0th order.

The initial conditions (64) give $f(m, t)=\frac{1}{2 m_{0}} G\left(m, m_{0}, t\right)$.

One can easily show that

$$
\frac{\partial}{\partial m} Z_{\lambda}\left(m, m_{g}\right)=\frac{2}{\pi m_{g}} \frac{1}{\left(J_{0}^{2}\left(\sqrt{\lambda} m_{g}\right)+Y_{0}^{2}\left(\sqrt{\lambda} m_{g}\right)\right)^{1 / 2}} .
$$

Then the flux

$$
\begin{aligned}
& S_{J}(t)=\left.m_{g} R \frac{1}{2 m_{0}} \int_{0}^{\infty} d \lambda m_{0} \exp (-\lambda R t) Z_{\lambda}\left(m_{0}, m_{g}\right) \frac{\partial Z_{\lambda}\left(m, m_{g}\right)}{\partial m}\right|_{m=m_{g}}= \\
= & \frac{R H(x, y)}{\pi R t}, H(x, y)=\int_{0}^{\infty} d \eta \exp (-\eta) \frac{J_{0}(\sqrt{\eta} x) Y_{0}(\sqrt{\eta} y)-J_{0}(\sqrt{\eta} y) Y_{0}(\sqrt{\eta} x)}{J_{0}{ }^{2}(\sqrt{\eta} x)+Y_{0}^{2}(\sqrt{\eta} x)} .
\end{aligned}
$$

Here we have changed the variables: $\eta=\lambda R t, x=m_{g} / \sqrt{R t}, y=m_{0} / \sqrt{R t}$.

One can show that $H(x, y) \simeq Z(x, y) \exp \left(-\zeta y^{2}\right)$ at $y \geq x+4, Z(x, y)$ weakly depends on its arguments, $\zeta \sim 5$. Hence $S_{J}(t) \propto \frac{1}{t} \exp \left(-\frac{m_{0}{ }^{2}}{\zeta R t}\right)$. To obtain exact form of the dependence, we take the following consideration.

The flux $S_{J}(t, m)=m R \frac{\partial f}{\partial m}$ is a continuous function of $m$; in the region $m_{g}<$ $m<m_{0}$ we may take it to be a constant independent of $m$ (it is correct for values of $t$ greater than certain $t_{0}$ when the width of peak of DF becomes comparable with $m_{0}$, see fig 2). Let us denote $\kappa(t)=\left.\frac{\partial f(m, t)}{\partial m}\right|_{m=m_{g}}$, then we have

$$
S_{J}(t)=m_{g} R \kappa(t), \quad f(m, t)=\int_{m_{g}}^{m} \frac{S_{J}}{R m^{\prime}} \partial m^{\prime}=m_{g} \kappa(t) \ln \frac{m}{m_{g}} .
$$

Thus we obtain that $f(m, t)$ grows logarithmically with $m$. Its dependence on time can be found from the argument that $\kappa(t) \approx \frac{\Xi}{t} \exp \left(-\frac{m_{0}{ }^{2}}{5 R t}\right), \Xi$ is a constant.(fig. 3). $\kappa(t)$ reaches maximal value at the exponent argument equal to -1 ; 
its maximal value $K_{\max }=\frac{\Xi 5 R}{\mathbf{e} m_{0}^{2}}$. To determine the value of $\Xi$ we apply expression (66) for $m=m_{0} / 2$; having found analytically $f_{\max }\left(m_{0} / 2\right)=\frac{\ln 2}{2 m_{0}{ }^{2}}$, we obtain $\Xi=\frac{\mathbf{e} \ln 2}{10 m_{g} R \ln \frac{m_{0}}{2 m_{g}}}$. Finally, the flux is

$$
S_{J}(t)=\frac{\mathbf{e} \ln 2}{10 \ln \frac{m_{0}}{2 m_{g}}} \cdot \frac{1}{t} \exp \left(-\frac{m_{0}^{2}}{5 R(J) t}\right)
$$

The correctness of the above consideration is proved by numerical investigation of the problem. We also should notice that, in fact, expr. (67) slightly overestimates the flux at $t \geq \frac{m_{0}^{2}}{R(J)}$ (up to a factor of 1.5 , see fig. (4).

\subsection{Black hole growth law}

Firstly, we neglect that in central region of Coulomb potential the coefficient $R(J)$ differs from the expression (51) and take $R(J)=R_{\epsilon} J^{\epsilon}, \epsilon=\frac{\alpha}{2+\alpha} \leq 0.2$. Substituting the obtained value (67) of $S_{J}(t)$ into the expression (63) for total flux, we rewrite it as follows:

$$
S(t)=\int_{0}^{\infty} f_{0} J^{1 / 8} \frac{0.18}{\ln \frac{l_{0} J}{2 m_{g}}} \frac{1}{t} \exp \left(-\frac{l_{0}^{2} J^{2-\epsilon}}{5 R_{\epsilon} t}\right) .
$$

The absorption boundary $m_{g}$ changes with time. However, the flux weakly depends on the value $m_{g}$. We approximate the logarithm in the denominator to have a constant value $\sim 10$.

$$
S(t)=8.9 f_{0} H_{\epsilon} \frac{R_{\epsilon}^{\frac{9}{8(2-\epsilon)}}}{t^{1-\frac{9}{8(2-\epsilon)}}}, \quad H_{\epsilon}=\left(\frac{5}{l_{0}^{2}}\right)^{\frac{9}{8(2-\epsilon)}} \frac{\Gamma\left(\frac{9}{8(2-\epsilon)}\right)}{2-\epsilon} .
$$

Supposing that black hole growth is governed only by the absorption of dark matter, we obtain the following growth law for $\mathrm{BH}$ mass (assuming the mass of the seed black hole to be small):

$$
M_{b h}(t)=B\left(R_{\epsilon} t\right)^{\frac{9}{8(2-\epsilon)}}, \quad B=8.9 f_{0} H_{\epsilon} \frac{8(2-\epsilon)}{9}
$$

Notice that more precise expression for $S_{J}(t)$ at large $t$ reduces the value of $B$ approximately 1.2 times.

Thus the black hole growth is power-law with power index about $9 / 16$. This is in good agreement with previous work 7 , though the power index is a bit lower.

\subsection{Influence of central region onto the black hole growth}

One could suppose that the diffusion goes slower in the central region since the diffusion coefficient is lower there, and it may affect total growth law. In fact, nevertheless, it is not true. As has been shown in 3.3 the characteristic timescale for diffusion $\tau \propto M_{b h}{ }^{2}$ and is about $10^{6} \mathrm{yr}$. at the moment, hence it was even lower previously. Additionally, from (67) one can see that maximum of the flux goes from 
inside the region $J_{\max } \leq \sqrt{5 R t / l_{0}^{2}}$, which at the moment corresponds to spatial area $r \leq 100 \mathrm{pc}$, and value of $J_{0}$ separating the central and outer parts of the bulge corresponds to $r \simeq 1$ pc. These two quantities depend on time in similar ways: $J_{\max } \sim t^{1 / 2}, J_{0} \sim M_{b h}(t) \sim t^{9 / 16}$, so the relation $J_{0} \ll J_{\max }$ was also true in the past.

One can show that if $R$ varies in time, then we should take the following expression for $S_{J}(t)$ :

$$
S_{J}(t)=\frac{\mathbf{e} \ln 2}{10 \ln \frac{m_{0}}{2 m_{g}}} \cdot \frac{R(t)}{\int_{0}^{t} R\left(t^{\prime}\right) d t^{\prime}} \exp \left(-\frac{l_{0}^{2} J^{2}}{5 \int_{0}^{t} R\left(t^{\prime}\right) d t^{\prime}}\right)
$$

For each $J$ we define $t_{\max }(J)$ to be the time of maximal flux, and $t_{0}(J)$ to be the time to enter the BH domain of influence: $J_{0}\left(t_{0}\right)=J$. The diffusion coefficient $R(t)$ starts to decrease after the time $t_{0}$. But one can easily see that $t_{0}(J) \simeq 10^{4} \frac{l_{0}^{2} J^{2}}{(5 R)}=$ $10^{4} t_{\max }(J)$, from which we conclude that this correction affects only the far "tail" of $S_{J}(t)$, when then most part of the flux is already absorbed, and is practically unimportant for growth law.

\subsection{Influence of two-dimensional diffusion onto the black hole growth}

Now let's try to estimate the effect of diffusion along $J$ axis, i.e. the correctness of one-dimensional approximation. We restrict our consideration to the case $\alpha=$ 0 when $R_{22}=$ const. Having obtained approximate solution for one-dimensional diffusion equation. we substitute it into initial equation (17) and calculate the first term

$$
\eta=\frac{\partial}{\partial J}\left(R_{11} \frac{\partial f}{\partial J}\right)=\frac{\partial}{\partial J}\left(R_{22} 0.1 \frac{J}{m} \frac{\partial}{\partial J}\left\{\frac{\mathbf{e} \ln 2}{10 \ln \frac{l_{0} J}{2 m_{g}}} \frac{\ln \frac{m}{m_{g}}}{R_{22} t} \exp \left[-\frac{l_{0}^{2} J^{2}}{5 R_{22} t}\right]\right\}\right) .
$$

Of special interest is the time $t \sim t_{\max }$ corresponding to the maximum of the flux: $t_{\text {max }}=\frac{l_{0}^{2} J^{2}}{5 R}$. It appears that $\eta \propto t_{\max }-t$ :

$$
\eta=\frac{\ln 2}{5 \ln \frac{l_{0} J}{2 m_{g}}} \frac{R_{22} \ln \frac{m}{m_{g}}}{l_{0}^{2} J^{3} m}\left(1-\frac{t}{t_{\max }}\right) .
$$

Now we should compare $\eta$ with the second term in (17) $\eta_{m}=\frac{1}{m} \frac{\partial}{\partial m}\left(m R_{22} \frac{\partial f}{\partial m}\right)$. However, one can easily see that substituting (66) into this term makes it zero, since this approximate solution does not satisfy initial equation in the whole region $m>m_{g}$. To avoid this problem, we calculate the left-hand side of (59) $\eta_{m}=\frac{\partial f}{\partial t}$ :

$$
\eta_{m}=\frac{5 \ln 2}{2 \ln \frac{l_{0} J}{2 m_{g}}} \frac{R_{22} \ln \frac{m}{m_{g}}}{l_{0}^{4} J^{4}}\left(1-\frac{t}{t_{\max }}\right) .
$$

Comparing with (71) we obtain $\frac{\eta}{\eta_{m}}=\frac{2 l_{0}^{2} J}{25 m}$. 
For $J\left(r_{+}=100 \mathrm{pc}\right), m=m_{g}$ this ratio is about 30 . However, it was shown at the end of 3.4 that to estimate maximal value of $R_{11}$ one should take $m \sim 10^{2} m_{g}$ instead of $m=m_{g}$, which lowers this ratio to about unity.

It should be emphasized that all these estimations are rather approximate, since for precise assertions one should know the exact solution of one-dimensional diffusion. The arguments of 4.2 give only the correct value for flux through $m=m_{g}$, but not the exact solution for all $m$.

In general case, the inapplicability of reduction to one-dimensional diffusion at $m \rightarrow m_{g}$ does not change much the value of flux through $m=m_{g}$, since the diffusion along $J$ axis leads to the "blur" of distribution function along this axis, while the coefficient $R_{22}$ only weakly depends on $J$ as follows from (53). This is a benefit of selecting radial action as the second variable instead of energy as it was in previous works 24. However, an especial consideration is necessary whether this diffusion may lead to the drift of dark matter into the region of large $J$ where changes of $m, J$ during one period exceed their values, and diffusion approximation becomes incorrect.

\section{Comparison with observations and conclusions}

In conclusion, we make theoretical estimations of black hole masses obtained from growth law (70) and compare them with observational data for several galaxies.

As it was noted previously, in distant galaxies it is difficult to measure precisely the dependence of velocity dispersion on radius. However, in galaxies M 31 and NGC 4258 it seems to be almost constant and equals approximately $200 \mathrm{~km} / \mathrm{s}$.26. Taking the value of $f_{0}$ the same as in our Galaxy (12) and the time of growth $t=3 \cdot 10^{17} \mathrm{~s}=10^{10} \mathrm{yr}$. we obtain the value $M_{b h}=1.8 \cdot 10^{7} M_{\odot}$. The observed black hole masses are $(2.0 \div 8.5) \cdot 10^{7} M_{\odot}$ for M 31 and $3.8 \cdot 10^{7} M_{\odot}$ for NGC 4258 [16. The comparison shows that dark matter may comprise significant fraction of black hole masses in these galaxies.

As for Milky Way, the rotation curve is not flat in the center of the bulge, and the velocity dispersion may be approximately represented as $\sigma(r)=\sigma_{0}\left(\frac{r}{10 \mathrm{pc}}\right)^{1 / 4}$, $\sigma_{0}=60 \mathrm{~km} / \mathrm{s}$ 16. This corresponds to the value $\alpha=0.5$. Using the assessment (12) for $f_{0}$ the expression (70) gives the black hole mass $M_{b h} \approx 10^{7} M_{\odot}$, which clearly overestimates the adopted value of $M_{b h} \approx 2.9 \cdot 10^{6} M_{\odot}{ }^{20}$ (about a factor of three). Notice that these values are about twice as smaller as in Ref. 7, because of more precise estimate of dark matter flow (69). If we take $\sigma=$ const $=100 \mathrm{~km} / \mathrm{s}$, the numbers change only a little.

The disagreement with observations may be explained by rather rough estimates of quantities $f_{0}$ and $l_{0}$, which are determined by the whole dark matter halo and are different for different galaxies. If we take the value for DM fraction in bulge $\Upsilon \sim 0.24 \div 0.31 \frac{13}{13}$, then the resulting BH mass will be almost in agreement with observation. 
In conclusion, we can say that the model discussed can give reasonable estimate for observed masses of giant black holes in galactic centers. It is likely that a large fraction of black hole mass may be comprised of dark matter.

Further development of this problem will require, firstly, more precise calculation of diffusion coefficients based on detailed data for star distribution in the central parts of the bulge; secondly, taking into account the bulge evolution; and finally, an exact consideration of two-dimensional diffusion accounting for particles Fermiheating and their drift out of bulge.

The authors are grateful to A.V. Gurevich, K.P. Zybin, A.S. Ilyin and V.A. Sirota for numerous fruitful discussions. This work was supported by RFBR grants 01-02-17829, 03-02-06745, Russian Ministery of Education and Science grant 2063.2003.2, and the Forschungszentrum Juelich grant within the framework of the Landau Program.

\section{References}

1. P. Gondolo, J. Silk Phys.Rev.Lett. 83, 1719 (1999).

2. J. D. MacMillan, R. N. Henriksen Astrophys.J. 569, 83 (2002).

3. P. Ullio, H. Zhao, M. Kamionkowski Phys.Rev. D64, 043504 (2001).

4. J. Navarro, C. Frenk, S. White ApJ 490, 493 (1997).

5. A. Gurevich Proc. III ${ }^{r d}$ International Sakharov Conference (2002).

6. K. Zybin, A. Ilyin Proc. III ${ }^{r d}$ International Sakharov Conference (2002).

7. A. Ilyin, K. Zybin, A. Gurevich Journal of expreimental and theoretical physics, 98, No. 1, 1 (2004); astro-ph/0306490

8. A.V. Gurevich, K.P. Zybin Uspekhi fiz. nauk 165, No.7 (1995).

9. L. Fukushige, J. Makino, ApJ 477, L9 (1997).

10. B. Moore et al. MNRAS 310, 1147 (1999).

11. L.L.R. Williams, A. Babul, J. Dalcanton, Astrophys.J. 604, 18 (2004).

12. A. Klypin, A. Kravtsov, J. Bullock, J. Primack, Astrophys.J., 554, 903 (2001).

13. A. Klypin, H. Zhao, R. Somerville; Astrophys.J. 573, 597 (2002).

14. J. Navarro astro-ph/0311361

15. M. Bureau ASP Conference series, Vol.III, 2002; astro-ph/0203471

16. S. Tremaine, K. Gebhardt et al., Astrophys.J. 574, 740 (2002).

17. J. Kormendy, K. Gebhardt astro-ph/0105230

18. R. Genzel astro-ph/0008119

19. T. Ott, R. Schrödel, R. Genzel et al. The Messenger 111, 1 (2003); astro-ph/0303408

20. R. Schrödel, R. Genzel, T. Ott, A. Eckart Astron. Nachr., 324, No. S1 (2003), Special Supplement "The central 300 parsecs of the Milky Way"; astro-ph/0304197

21. W. Dehnen, J. Binney MNRAS 294, 429 (1998).

22. E.M. Lifshits, L.P. Pitaevskii "Physical Kinetics", Pergamon Press, Oxford, 1981.

23. J. Bahcall, R. Wolf ApJ 209, 214 (1976).

24. A. Lightman, S. Shapiro ApJ 211, 244 (1977).

25. S.M. Faber, S. Tremaine, E. Ajhar et al., Astron.J. 114, 1771 (1997).

26. Y. Sofue, V. Rubin Ann. Rev. Astron. Astrophys. 39, 137 (2001).

27. J. Silk Int.J.Mod.Phys. A17(Suppl.1), 167 (2002); astro-ph/0110404

28. W. Saslaw "Gravitational physics of stellar and galactic systems", Cambridge Univ. Press, 1987. 


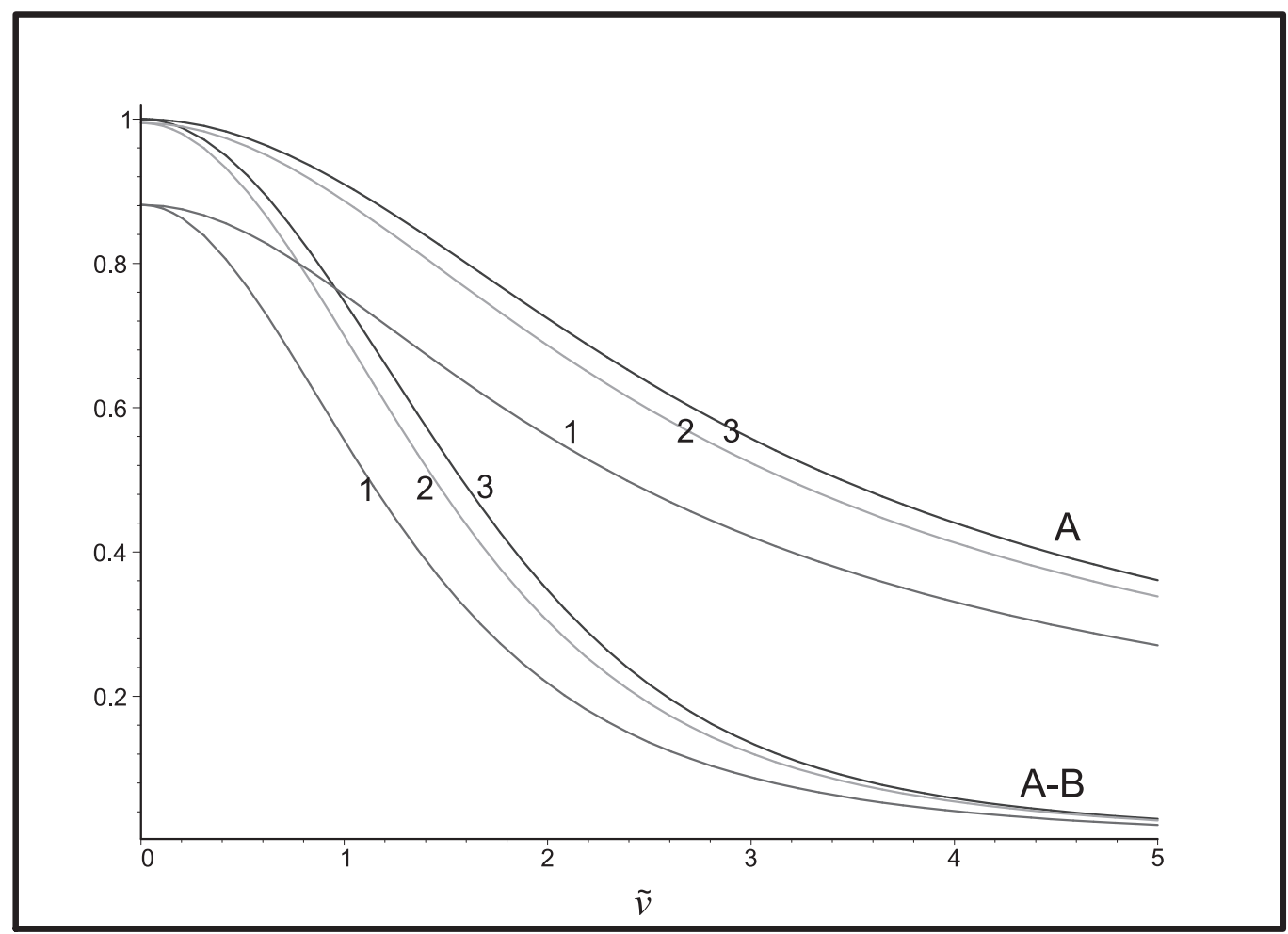

Fig. 1. The graphs of functions $\widetilde{A}(\tilde{v})$ (coefficient $A$, 48), $\widetilde{\Omega}(\tilde{v})$ (coefficient $A-B$, 48 ) for different potentials: 1) power-law $\left.\left(\Psi \propto r^{\alpha}\right), \alpha=1 / 2 ; 2\right)$ power-law, $\left.\alpha=1 / 4 ; 3\right)$ isothermal $(\alpha=0, \Psi \propto \ln r)$. 


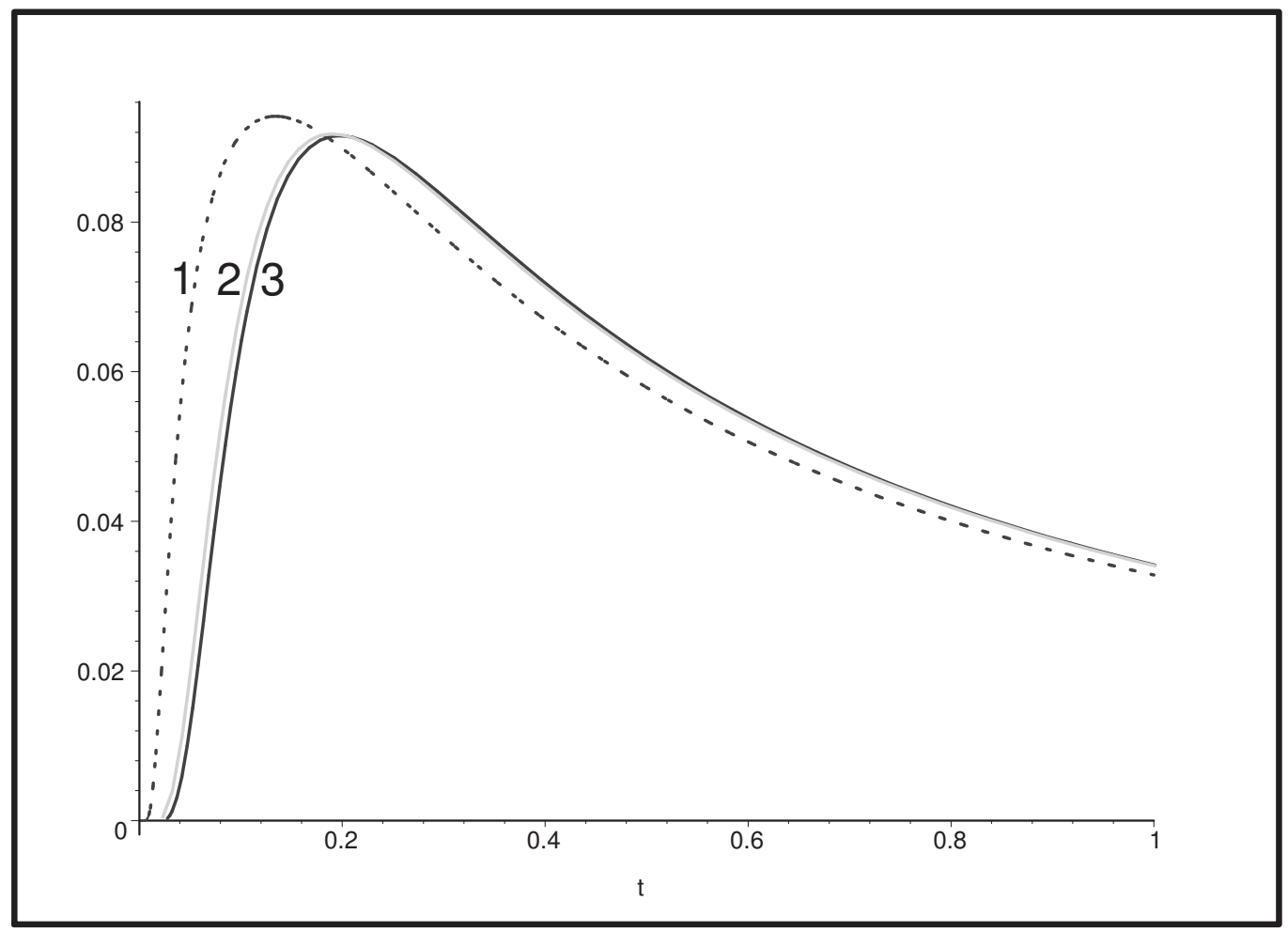

Fig. 2. Normalized values $f(m, t) / \ln \left(m / m_{g}\right)$ for different $m$ [66: 1) $\left.m=m_{0} / 2,2\right) m=20 m_{g}$, 3) $m=1.2 m_{g}$. One can see that the graphs are quite similar, the normalized maximal values are approximately equal, though they are reached at different $t$.

The values are given for $m_{0}=100 m_{g}$. 


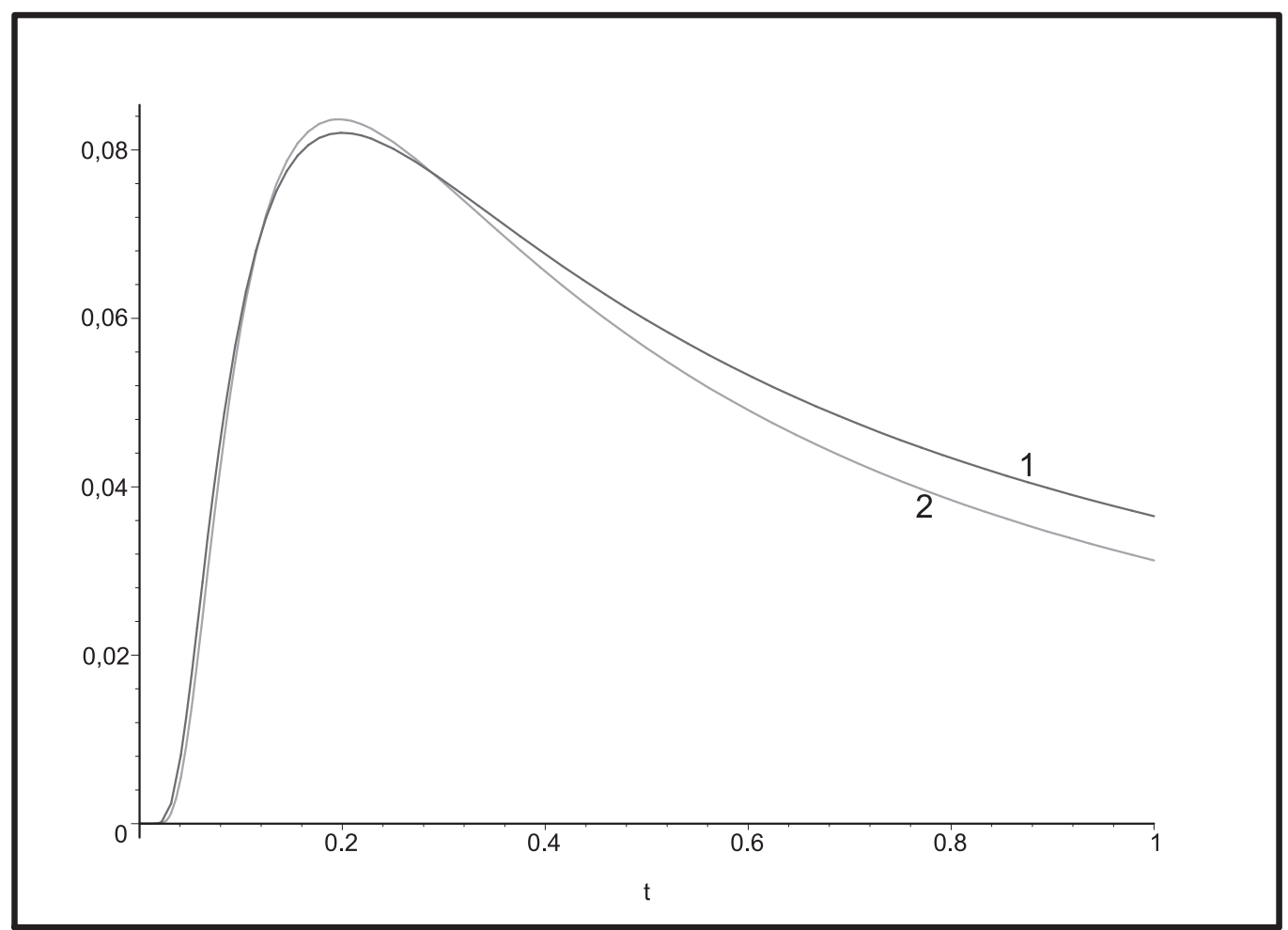

Fig. 3. The flux function $\kappa(t): 1)$ theoretical [67, 2) obtained by numerical integration. One can see that the theoretical approximation is good enough for maximum of the flux, but overestimates it at large $t$. 


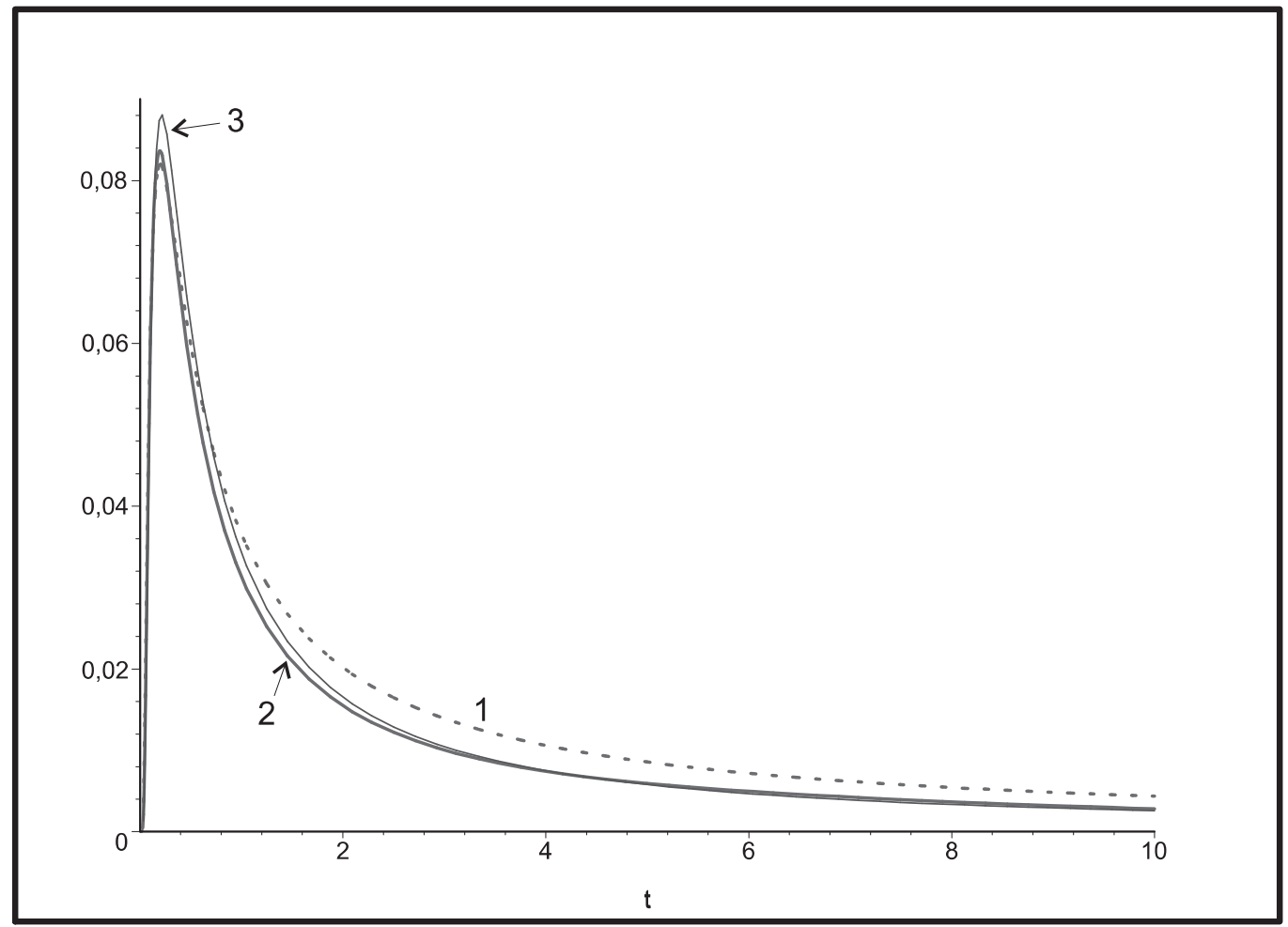

Fig. 4. The corrected flux function $\kappa(t)$ for large $t: 1)$ theoretical function (fig. 3), 2) obtained by numerical integration, 3) correcter theoretical function: $\kappa \propto t^{-5 / 4}$. The last approximation is much better. 